Isak Potgieter

\title{
Privacy concerns in educational data mining and learning analytics
}

\begin{abstract}
:
Education at all levels is increasingly augmented and enhanced by data mining and analytics, catalysed by the growing prevalence of automated distance learning. With an unprecedented capacity to scale both horizontally (individuals reached) and vertically (level of analysis), data mining and analytics are set to be a transformative part of the future of education. We reflect on the assumptions behind data mining and the potential consequences of learning analytics, with reference to an issue brief prepared for the U.S. Department of Education entitled Enhancing Teaching and Learning Through Educational Data Mining and Learning Analytics. We argue that the associated gains conceal subtle, but important risks. Data-ism, an underpinning paradigm, assigns unjustified veracity to data-driven science and the application of personalised analytics may compromise individual privacy, agency and inventiveness. This holds serious ethical implications, particularly when considering the impact on minors, rendering wholesale adoption premature.
\end{abstract}

\section{Keywords:}

Analytics, Big Data, Data Mining, Data, Education, Learning, Privacy

\section{Outline:}

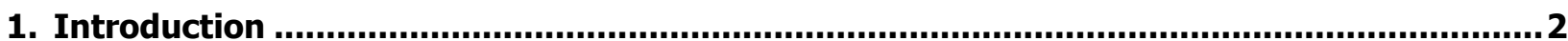

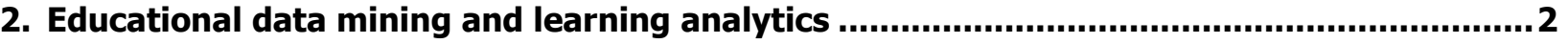

3. Educational data mining's empirical underpinnings ................................................ 2

4. Learning analytics and its bearing on autonomy .........................................................

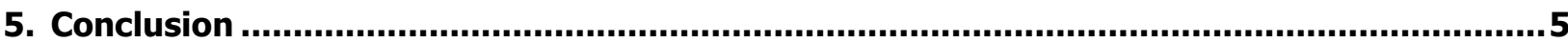

6. References.............................................................................................................. 6

\section{Author:}

Isak Potgieter:

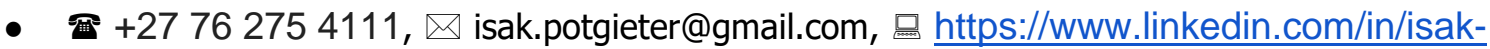
potgieter-52b50512/ 


\section{Introduction}

An issue brief prepared for the U.S. Department of Education in 2012 by the Center for Technology in Learning entitled Enhancing Teaching and Learning Through Educational Data Mining and Learning Analytics (Bienkoswki et al.) promotes enthusiastic adoption of the mechanisms involved in an emergent "fourth paradigm in science" (Kitchen) and an affirmation of its claims. "The goal of creating an interconnected feedback system would be to ensure that key decisions about learning are informed by data and that data are aggregated and made accessible at all levels of the education system for continuous improvement" (Bienkoswki et al.). This essay outlines the ways in which the proposed system aims to utilise data processing to inform all aspects of educational practice and considers the efficacy of its underlying assumptions and their ethical implications.

\section{Educational data mining and learning analytics}

The brief broadly divides the activities involved in its recommendations into two domains, educational data mining and learning analytics. These are over- lapping and mutually informative, but their salient distinctive features as explained in the report include the following. Educational data mining aims to discover meaningful educational patterns by taking an experimental approach, using techniques from statistics, machine learning and data mining to analyse data collected during teaching and learning to understand how students learn, predict future student learning behaviour and test hypotheses. As models are tested and the effects of different pedagogical tools are measured over time, the models are in turn improved. This process ultimately advances educational knowledge and informs practice.

Learning analytics then focuses on pattern discovery in individual behaviour using established instructional methods and models. The methods used include those of data mining and machine learning as well as peripheral tracking like social network analysis and attention metadata based on user engagement and involves techniques from a broader spectrum, including information science, sociology and psychology. It monitors and predicts students' progress, identifies where intervention may be required and determines the best arrangement of courses for an individual. It aids human tailoring of responses that directly influence educational practice. Thus, educational data mining develops new tools for pattern discovery and learning analytics applies those and other tools in educational practice.

\section{Educational data mining's empirical underpinnings}

The system described by the report is one that ambitiously collects as much data as possible about the methods used and the way learners engage with them. In general its modus operandi is in line with the methodology of data-ism (Kitchen) which sees the world "in a way that asserts everything is data" (Sadowski), that data are "natural and essential elements that are abstracted from the world in neutral and objective ways and can be accepted at face value" allowing big data to potentially "capture the whole of a domain and provide full resolution" (Kitchen). Cohen states that "Big Data is the ultimate expression of a mode of rationality that equates information with truth and more information with more truth" (Cohen).

These premises are increasingly pervasive in the burgeoning era of Big Data as the 'data revolution' systematically transforms the way in which much of commercial and scientific enterprise is conducted. If data analytics has the capacity to discover truth in high fidelity, then the rapid development of datadriven -as distinct from hypothesis-driven-research and the self-reinforcing nature thereof mean that we are on a trajectory of unprecedentedly rapid discovery. Given that improved comprehension of the 
world is beneficial, then to the extent that these claims are accurate such exploration will on balance be eminently desirable.

Data do not, alas, come to us neatly packaged and labelled, but have to be contextualised and interpreted. Sadowski challenges the neutrality of observations since they "do not merely reveal or reflect the world [but] order and construct the world" (Sadowski). At every stage of data processing bias is introduced. Kitchin contends that while data-ism strives to exhaustively capture the whole of a domain, it is viewed from a vantage point and is therefore oligoptic, not panoptic. "Data do not pre-exist their generation and arise from nowhere. Rather data are created within a complex data assemblage that actively shapes its constitution" (Kitchin). Therefore, algorithms inevitably make biased decisions, reflecting its designer and intended uses (Mittelstadt) and (Cohen) Learning requires data and the accumulation of it will undoubtedly enlighten, but data-ism may be a construction in need of Kant's Copernican Revolution.

Machine learning itself, currently spearheaded by deep learning, may embody some fundamental limitations. A challenge facing machine learning is the inability to inherently distinguish causation from correlation (Marcus). That the two are not identical is a mantra of basic statistics, but as if the disclaimer entitled correlation to account for both, questions about causality were abandoned by statistics. Questions about cause-and-effect relationships that are common in everyday language lack basic scientific articulation. Much of statistics and its derivatives have remained in what Pearl calls the "Prohibition era" of causality and thus believes that data alone can answer scientific questions by way of sophisticated data mining. The lack of causal comprehension in machines is a serious challenge to the efficacy of Big Data as the key to continued discovery of the world.

The contention here is not that educational data mining will result in interpretive deterioration, on the contrary, but that it is not the superconductor of truth that some of its proponents believe. Given the foundational impact of such a system on a child's formative years, we should be hesitant to put complete faith in the efficacy of data-driven science.

\section{Learning analytics and its bearing on autonomy}

The issue brief emphasises the extent to which all aspects of a learner's activity will be recorded. In addition to learning activities, learning analytics will employ methods to do an analysis of "student-tostudent and student-to-teacher relationships and interactions" on social networks and also collect "attention metadata" to determine what a user is interacting with. This will be used to do user modelling, comprising learner understanding, behaviour, motivation, experience and level of satisfaction (Bienkoswki et al.). Such comprehensive coverage will most likely improve the achievement of educational goals but will do so in exchange for personal data. It leads to a range of privacy considerations, some of which will be outlined here. Making provision for some scope for legitimate surveillance of minors by parents and educators, the task is to determine the extent to which ethical considerations of privacy as applied to adults are relevant here as well.

The scope of learning analytics extends far beyond what was previously possible both in breadth (including extracurricular activity) and depth (recording individual action in as much detail as possible), representing a form of dataveillance (Kitchin). This prompts consideration of the role of consent in the collection and use of personal information. The importance of well-informed consent has received much attention (Mai) in ethical literature, which in the case of minors is the responsibility of parents or legal guardians. Minors are already legitimately monitored by guardians and educators offline, but the level of monitoring described in the brief is perhaps currently only exceeded by state surveillance like that in China. The full implications for privacy are hard to grasp. 
The brief champions the ability of these methods to predict future behaviour based on patterns in learner data. "These online or adaptive learning systems will be able to exploit detailed learner activity data not only to recommend what the next learning activity for a particular student should be, but also to predict how that student will perform with future learning content, including high-stakes examinations" (Bienkoswki et al.). The extraction of this new predictive layer calls for a shift in thinking about privacy to consider what Mai calls a "datafication model" of privacy, which, in addition to established surveillance and capture privacy models, is needed when considering data-driven practices (Mai). Where the traditional models focus on the collection of data and their use, the datafication model shifts the focus to data processing and analysis. It assumes that data have been collected and is concerned with the construction of "new insights generated from available data". This is a dimension that is unlikely to be considered by parents and implies that rather than statically populating storage space, data collected during formative years will dynamically live on.

Another characteristic of central data collection is its imperishability and consequential capacity for dissemination. The data trail is not discarded with the passage time nor is access to it limited to the handful of individuals who have primary or secondary contact with a learner (parents, teachers, headmasters, psychologists). The brief indicates provisions made by the Family Educational Rights and Privacy Act (FERPA) for the disclosure of "personally identifiable information from a student's education record without consent to 'school officials' if there is a legitimate educational interest" (Bienkoswki et al.). Such access is desirable to the extent that it is granted only for the intended purpose, but it bestows inordinate power on the judges of legitimacy. Well-connected individuals may not face the same hurdles as educators. If abused, this system would present a potent instrument for the concentration of power, where information unjustly gained could be used as political or legal leverage or amalgamated into additional databases for further profiling and targeting. So much for the right to be forgotten. Flawed (or even subsequently unfashionable) childhood behaviour may forever haunt future generations. It is hard to imagine that this formulation would be accepted in any liberal democratic environment were it applied to adults.

As learners become aware of the reach of dataveillance, it is likely to lead to self-censorship and a reluctance to take risks. Learners may become disinclined from even engaging in benign activities in order to avoid notice. Such "'chilling effects' are at odds with human rights and democratic practice and can lead to behavioural uniformity and a stifling of creativity" (Macnish). Kupfer states that "we need privacy to try out options and rehearse our thinking" (Weston). This is a crucial part of personal development. Constant supervision is likely to interfere with character development, which relies on a certain level of experimentation. "The autonomous person, in developing character, will be aware of choices and their lasting impact on his or her life [which] is defeated with the enduring compilation of data about people" (Weston 2016). Critically, surveillance also diminishes the value of unsupervised play. Gray connects a decline in unsupervised play time with the rise in psychopathology. Gray actually viewed the internet as children's "saving grace" and the last bastion of freedom where they continue to "maintain their privacy" (Gray). Not anymore.

One of the most promising aspects of online learning in general is its ability to personalise the learning experience. By considering prior performance behavioural characteristics, course requirements and feedback, the selection of content, learning tempo, task sequence and other contextual variables can be tailored to suit each learner's needs and preferences. Insofar as personalisation provides for learning diversity it may solve the problem of curricular conformity so stifling to idiosyncratic youngsters in a way that has been infeasible until now. It will transform teaching from a top-down to bottom-up approach and make it resemble the variation in the subjects it aims to serve.

This, however, is the bullseye. Missing it entails significant implications. The risks of personalisation are much more subtle, but insidious, due to its invasive- ness. One of which is the constraining factor of learning objectives. It is a legitimate feature of the system, but its interplay with personalisation is one which may have serious implications for student autonomy, a subset of privacy (Weston). Personalisation customises content in a way that is informed by individual preferences, allowing them to dictate the 
framing of the learning environment to some extent. An important component enabling this is predictive modelling that anticipates future behaviour and initiates corresponding sequences of events. It is thus highly customisable, but with a particular goal in mind, which implies that guidance will always be involved. The brief does not shy away from its vision of continuous coaxing. "At the simplest level, analytics can detect when a student in an online course is going astray and nudge him or her on to a course correction. At the most complex, they hold the promise of detecting boredom from patterns of key clicks and redirecting the student's attention" (Bienkoswki et al.). This has paradoxical implications for the decisional freedom of learners. "A system that seeks to make people act on impulses or temptations and pre-empt the control process people can develop to resist impulses, interferes with self-governance" (Weston 2016).

Cohen calls this mode of intervention 'modulation' (Cohen). "Modulation is a mode of privacy invasion, but it is also a mode of knowledge production designed to produce a particular way of knowing and a mode of governance designed to produce a particular kind of subject". Predictive analytics narrows down the optional spectrum according to its model of a learner's characteristics and steers them along an anticipated path. "If the prediction is correct, all should be well. But if the prediction is incorrect, or the person creates a new choice not in line with past conduct, then that rational choice (if it is a rational choice) is constrained or pre-empted" (Weston). Learners are free to do as they please, but not to develop what it is that they please, a condition that Cohen ominously calls 'constructed subjectivity'. "The techniques of Big Data subject individuals to predictive judgments about their preferences, and the process of modulation also shapes and produces those preferences." Insightfully, Cohen traces the effect this will have on innovation, an important economic driver, increasingly understood as "the absence of regulatory constraint" in policy discourse. Transparency has been targeted as a threat to intellectual property and innovation, but Cohen indicates that "it is modulation, not privacy, that poses the greater threat to innovative practice". The freedom to explore increases the likelihood of injury or failure, but also renders a clearer picture of the landscape with all its obstacles. This is fertile ground where "innovation emerges from the interplay between freedom and constraint". It becomes clear why "intellectual regimentation" poses a significant threat to innovative practice. "It thrives most fully when circumstances yield serendipitous encounters with new resources and ideas and afford the intellectual and material breathing room to experiment with them" (Cohen).

In light of an aggregate gain it may be argued that the value of supporting anomalous behaviour is overstated. Surely the lofty goal of an optimised education system simultaneously individually tailored justifies the potential curbing of creative tinkering. Granted, we are considering the impact on a small number of outliers, but that denies the outsized role of anomalous individuals in the trajectory that brought us is key to taking us forward. This is no less than a gamble on the forming of future Newtons and Einsteins.

\section{Conclusion}

The report on 'Enhancing Teaching and Learning Through Educational Data Mining and Learning Analytics' is an exemplar of a more general trend of datafication, where reality is increasingly comprehended via the collection and pro- cessing of large amounts of data. Its plans express much optimism about the ways in which data-driven learning can advance educational practice. Despite the promise it holds, as argued here there is serious cause for pause before implementing its methodology in full. Assertions to maximal accuracy of knowledge claims cannot justifiably be maintained and the transformative impact that it will have on the autonomy of learners is cause for concern. The premise that "more information is better, more digital services improve people's lives, and greater connectivity provides a better world" requires revision. As Mason soberly observed some time ago: "our society is truly an information society, our time an information age. The question before us now is whether the kind of society being created is the one we want" (Mason). 


\section{References}

Bienkowski, M., Feng, M., and Means, B., "Enhancing teaching and learning through educational data mining and learning analytics: An issue brief," US Department of Education, Office of Educational Technology, pp. 1-64, 2012.

Cohen, J.E., "What privacy is for," Harvard Law Reverence., vol. 126, pp. 1904-1933, 2012.

Gray, P., "The decline of play and the rise of psychopathology in children and adolescents." American Journal of Play, vol. 3, no. 4, pp. 443-463, 2011.

Gray, P., "The culture of childhood: We've almost destroyed it," E-Journal of Psychology Today: Freedom to Learn, 2016

Kitchin, R., "The data revolution: Big data, open data, data infrastructures and their consequences". Sage, 2014.

Macnish, K., "Surveillance ethics," in Internet Encyclopedia of Philosophy, J. Fieser and B. Dowden, Eds., 2011.

Mai, J.E., "Big data privacy: The datafication of personal information," The Information Society, vol. 32, no. 3, pp. 192-199, 2016.

Marcus, G., "Deep learning: A critical appraisal," arXiv preprint arXiv:1801.00631, 2018.

Mason, R.O., "Four ethical issues of the information age," Mis Quarterly, pp. 5-12, 1986.

Mittelstadt, B.B., Allo, P., Taddeo, M., Wachter, S., and Floridi, L., "The ethics of algorithms: Mapping the debate," Big Data \& Society, vol. 3, no. 2, p. $2053951716679679,2016$.

Sadowski, J. "When data is capital: Datafication, accumulation, and extraction," Big Data \& Society, vol. 6, no. 1, p. $2053951718820549,2019$.

Weston, H., "Data analytics as predictor of character or virtues, and the risks to autonomy, "International Review of Information Ethics, vol. 24, no. 05, 2016. 